\title{
A SEARCH FOR HIGH EXCITATION NEBULAE AROUND WOLF-RAYET STARS IN THE MAGELLANIC CLOUDS
}

\author{
V. S. NIEMELA ${ }^{1 *}$, S. R. HEATHCOTE ${ }^{2}$ and W.G. WELLER ${ }^{2}$ \\ ${ }^{1}$ Instituto De Astronomia y Fisica del Espacio, CC67, Suc 28, 1428 Buenos Aires, \\ Argentina \\ ${ }^{2}$ Cerro Tololo Inter-American Observatory, National Optical Astronomy \\ Observatories", Casilla 603, La Serena, Chile
}

We have serendipitously discovered that the $\mathrm{WN}+\mathrm{O}$ binary $\mathrm{Br} 40 \mathrm{a}$ in the $\mathrm{LMC}$ is surrounded by a remarkable high excitation nebula, showing extended, narrow emission lines of HeII. This prompted us to make a systematic search for similar high excitation nebulae around other WR stars in the Magellanic Clouds. This surv ey revealed a second even more extreme example surrounding the $\mathrm{WN}+\mathrm{O}$ binary $\mathrm{AB}-7$ in the SMC, and one other marginal detection AB-5 (SMC). The detection of nebular HeII emission implies that these WN stars emit a much harder UV spectrum than is traditionally expected. For each of these nebulae we have taken narrow band CCD images at the CTIO 0.9-m telescope, and have obtained spectrophotometry with the 2D-Frutti photon counting detector on the CTIO 1.0-m telescope.

The WN3+O6 star $\mathrm{Br} 40 \mathrm{a}$ (Sk $\left.-71^{\circ} 34\right)$ is located in the outskirts of the bright HII region N206 in the LMC. Nebular HeII $4686 \AA$ line emission is detected in our two dimensional spectra over a region with an extent of 70 seconds of arc $(18 \mathrm{pc})$. This high excitation zone coincides with a partial ring shaped feature seen in a narrow band [OIII]5007 $\AA$ image. Figure 1 shows a one dimensional spectrum formed by averaging over the entire extent of the $\mathrm{He}^{++}$region.

The WN3+O6 star AB-7 (AzV 336a) is located near the centre of N76, the second brightest HII region in the SMC. A narrow band HeII $4686 \AA$ image shows an approximately circular high excitation zone 144 seconds of arc (37pc) in diameter, centred on the WR star. This $\mathrm{He}^{++}$region fills in a central hole in the annular nebula seen in a narrow band HeI $5876 \AA$ image. Figure 2 shows the average spectrum of the high excitation zone.

In our survey we found no evidence for nebular HelI emission in the vicinity of the 27 other WR stars, and 2 hot $\mathrm{O3}$ stars, which are listed in the table. This suggests that either only a small fraction of all WR stars reach such high temperatures, or that the high temperature phase is of extremely short duration, or that most WR stars modify their environment in such a way that a high excitation zone is undetectable.

\footnotetext{
"Visiting Astronomer CTIO, NOAO". Senior Visitor ESO. Member of Carrera del Investigador, CIC, Prov. Buenos Aires, Argentina. "CTIO, NOAO, operated by AURA Inc. under a cooperative agreement with NSF.
} 
Stars included in the survey for which nebular HeII $4686 \AA$ emission was not detected

Stars in the SMC

$\mathrm{AB} 1 \mathrm{WN3+OB}$

AB 2 Of/WN4.5

AB $3 \quad$ WN3+O4

AB 4 WN4.5

AB $6 \quad$ WN3+O7

AB 8 WO4+O4

\begin{tabular}{|c|c|c|c|c|c|}
\hline \multicolumn{6}{|c|}{ Stars in the LMC } \\
\hline Br 5 & Of/WN6+O7 & Br 37 & $\mathrm{WN} 3+\mathrm{OB}$ & Br 85 & WN4 \\
\hline $\mathrm{Br} 10 \mathrm{a}$ & Of/WN6 & Br 44 & WC4+OB & $\mathrm{Br} 93 \mathrm{a}$ & WN3 \\
\hline Br 16 & WN3 & Br 46 & WN4 & Br 95 & WN4+OB \\
\hline Br 23 & WN3 & Br 48 & $\mathrm{WN} 4+\mathrm{OB}$ & Br 99 & WN4 \\
\hline Br 25 & WN3 & Br 49 & $\mathrm{WN} 3+\mathrm{OB}$ & Br 100 & WN3 \\
\hline Br 26 & WN7 & Br 53 & WN4+OB? & Sk-71•51 & O3W(f*) \\
\hline Br 29 & WN3 & Br 66 & WN3 & Sk-67211 & O3M(f*) \\
\hline $\mathrm{Br} 31$ & WC4+O9 & $\mathrm{Br} 72$ & $\mathrm{~B} 1 \mathrm{I}+\mathrm{WN} 3$ & & \\
\hline
\end{tabular}

AB: numbers from Azzopardi and Breysacher (1979, Astron. Astrophys. 75, 120)

Br: numbers from Breysacher (1988, Astron. Astrophys. 160, 185)

Sk: numbers from Sanduleak (1970, CTIO contributions N289)

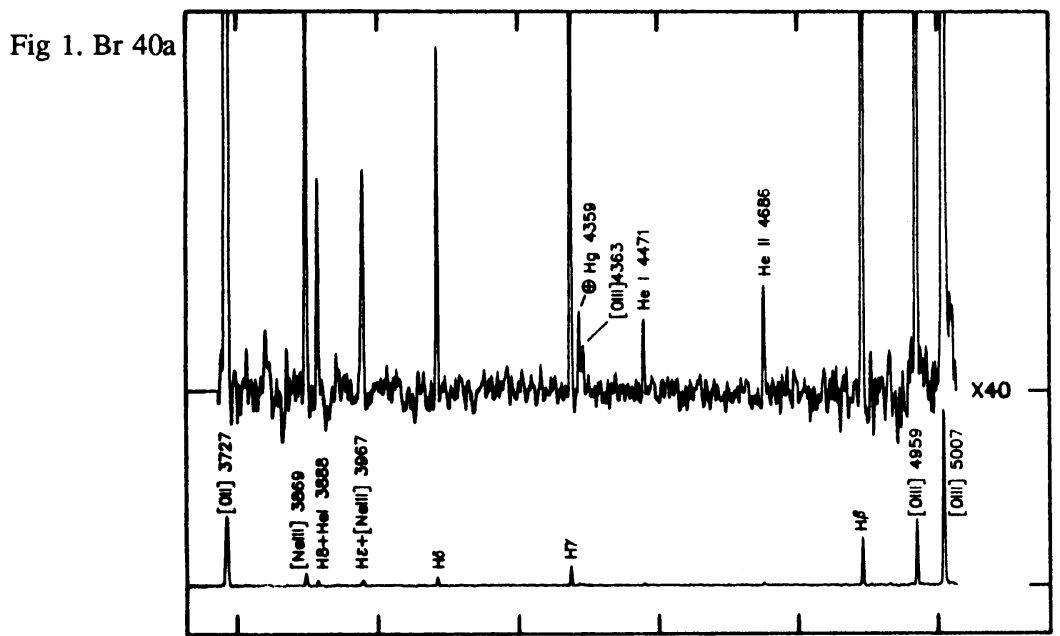

Fig 2. AB-7

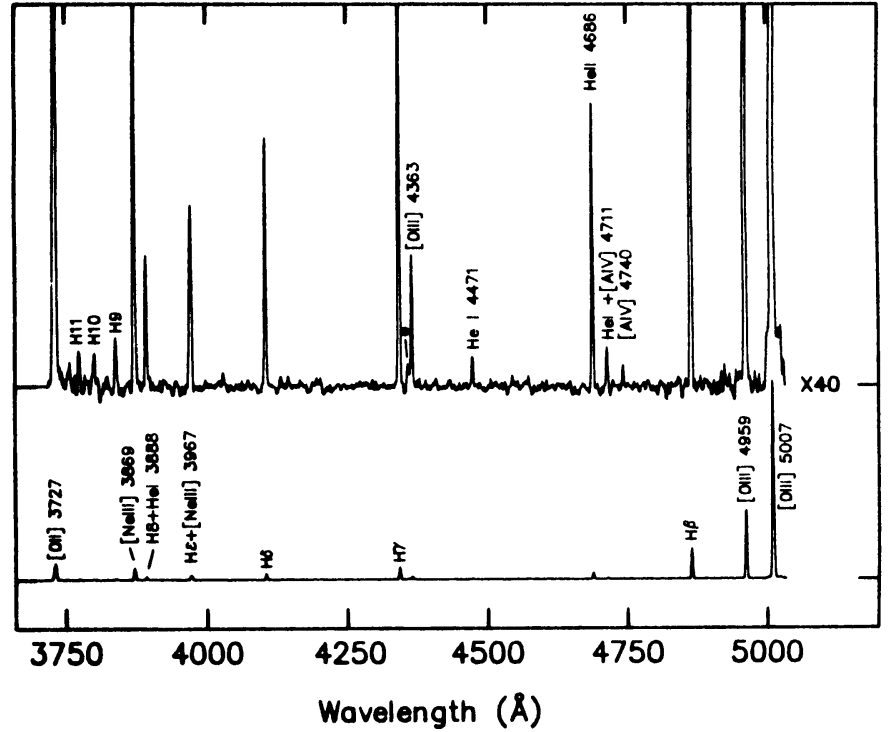

